\title{
In-Plane Stacking Disorder in Polydisperse Hard Sphere Crystals
}

\author{
Janne-Mieke Meijer, Volkert W. A. de Villeneuve, and Andrei V. Petukhov* \\ Van't Hoff Laboratory for Physical and Colloid Chemistry, Department of Chemistry, Faculty of Science, \\ Utrecht University, Padualaan 8, 3584 CH Utrecht, The Netherlands
}

Received October 9, 2006. In Final Form: November 22, 2006

\begin{abstract}
We demonstrate that in random-stacking hard-sphere colloidal crystals the stacking disorder not only exists in the direction perpendicular to the close-packed layers, but also extends in the lateral direction. The existence of such in-plane stacking disorder is suggested by a recent observation of lateral broadening of the Bragg scattering rods in microradian X-ray diffraction and is further confirmed here by real-space confocal microscopy in two hard-sphere colloidal systems with different relative gravity effects. Due to the in-plane stacking disorder, the hexagonal planes consist of islands with different lateral A, B, and C positions with characteristic line defects in between them. The real-space layer-by-layer stacks of images also reveal the 3-D structure of the defects. The chance $\xi$ to find another line-defect above a line-defect in the layer below turns out to be close to $1 / 2$-independent of relative gravity-which can be explained by the two different stacking options above a defect. The stacking of a few sets of several line defects situated on top of each other turns out to be predominantly FCC-like.
\end{abstract}

\section{Introduction}

Inherent to the natural ordering process of colloidal crystallization is the formation of defect structures, such as stacking faults, grain boundaries, and vacancies. The most efficient packing of spheres can be achieved by arranging them into stacked hexagonal close packed layers. ${ }^{1}$ Regular stacking sequences of $\mathrm{ABCABC}$ and $\mathrm{ABABAB}$ types lead to face-centered cubic (FCC) and hexagonal close-packed (HCP) structures, respectively. Although the FCC structure was proven to provide a little bit more space for particle fluctuations, the free-energy difference between FCC and HCP is extremely tiny, ${ }^{2-4}$ less than $10^{-3} k_{B} T$ per particle, where $k_{B} T$ is the thermal energy. As a result, a so-called random-hexagonal close-packed (RHCP) crystal structure is often found. ${ }^{5-9}$ Moreover, an additional gain of entropy due to the variety of possible RHCP configurations can stabilize the RHCP structure ${ }^{10}$ for sufficiently small crystals, although a flat wall seems to promote the FCC structure. ${ }^{11}$ Since crystal nuclei have RHCP structure and the reorganization from RHCP to FCC structure is expected to take months to years for experimental systems, ${ }^{8,12}$ RHCP structures are quite commonly observed in hard-sphere systems. The typical quantity of defects encountered is therefore much larger than that expected for equilibrium conditions.

The crystal structure can be conveniently characterized using diffraction techniques. ${ }^{9,13}$ In the reciprocal lattice of RHCP crystals, the reflections with $h-k$ not divisible by 3 are smeared

* Corresponding author: a.v.petukhov@chem.uu.nl.

(1) Sloane, N. J. A. Nature 1998, 395, 435-436.

(2) Bolhuis, P. G.; Frenkel, D.; Mau, S. C.; Huse, D. A. Nature 1997, 388, 235.

(3) Bruce, A. D.; Wilding, N. B.; Ackland, G. J. Phys. Rev. Lett. 1997, 79, 3002.

(4) Mau, S. C.; Huse, D. A. Phys. Rev. E 1999, 59, 4396.

(5) Pusey, P. N.; van Megen, W.; Bartlett, P.; Ackerson, B. J.; Rarity, J. G.; Underwood, S. M. Phys. Rev. Lett. 1989, 63, 1989.

(6) Zhu, J.; Li, M.; Rogers, R.; Meyer, W.; Ottewill, R. H.; Crew, S. -S. S.; Russel, W. B.; Chaikin, P. M. Nature 1997, 387, 883.

(7) Kegel, W. K.; Dhont, J. K. G. J. Chem. Phys. 2000, 112, 12.

(8) Dolbnya, I. P.; Petukhov, A. V.; Aarts, D. G. A. L.; Vroege, G. J.; Lekkerkerker, H. N. W. Europhys. Lett. 2005, 72, 962-968.

(9) Petukhov, A. V.; Dolbnya, I. P.; Aarts, D. G. A. L.; Vroege, G. J.; Lekkerkerker, H. N. W. Phys. Rev. Lett. 2003, 90, 028304.

(10) Auer, S.; Frenkel, D. Nature 2001, 409, 1020.

(11) Hoogenboom, J. P.; Vergeer, P.; van Blaaderen, A. J. Chem. Phys. 2003, $119,3371-3383$ out into so-called Bragg rods along the $l$ direction as a result of the stacking disorder. Here we use the usual RHCP basis, described in more detail in, for example, ref 9. However, since only three distinct lateral positions are involved, the stackingindependent reflections with $h-k$ divisible by 3 remain sharp. They correspond to periodicities common to all layers and will be referred to as Bragg spots. Figure 1a displays an example of a high-resolution small-angle X-ray scattering (SAXS) pattern obtained at the beam line BM-26 'DUBBLE' 14 of the European Synchrotron Radiation Facility (Grenoble, France) using microradian diffraction setup. ${ }^{13}$ The pattern is measured in a single colloidal crystal with an X-ray beam orthogonal to the hexagonal planes (i.e., $l=0$ ). The intensity of the diffraction peaks strongly decay with increasing length of the diffraction vector $q$ mostly due to the decay of the form factor. In addition, the structure factor of the Bragg spots (e.g., 110, 220, 300) is much stronger than that of the reflections originating from the Bragg rods (e.g., 100, 210).

Recently, microradian-resolution small-angle X-ray diffraction of a sedimented colloidal single-crystal revealed that the Bragg rods and Bragg spots have different widths also in the direction orthogonal to the $l .{ }^{13}$ Usually, one assumes that every layer has a unique lateral position and the stacking disorder is present only in the direction perpendicular to the close-packed layers. In that case both the stacking-dependent Bragg rods and the stackingindependent Bragg spots should be equally sharp in the lateral direction. The observed additional broadening of the Bragg rods within the $(h, k)$ plane can be understood if one assumes that the close-packed hexagonal (111) planes consist of islands with different $\mathrm{A}, \mathrm{B}$, or $\mathrm{C}$ lateral positions of the spheres with characteristic line-defects in between them as illustrated in Figure 1b. The stacking-independent Bragg spots, such as the 110 reflection, do not "notice" the difference between the islands, and therefore, they are not broadened by the island structure of the layers. In contrast, switching from one island to the other leads to an additional phase shift of $\pm 120^{\circ}$ of their contributions

(12) Pronk, S.; Frenkel, D. J. Chem. Phys. 1999, 110, 4589-4592.

(13) Petukhov, A. V.; Thijssen, J. H. J.; t Hart, D. C.; Imhof, A.; van Blaaderen, A.; Dolbnya, I. P.; Snigirev, A.; Moussaid, A.; Snigireva, I. J. Appl. Cryst. 2006, $39,137-144$.

(14) Bras, W.; Dolbnya, I. P.; Detollenaere, D.; van Tol, R.; Malfois, M.; Greaves, G. N.; Ryan, A. J.; Heeley, E. J. Appl. Cryst. 2003, 36, 791-794. 

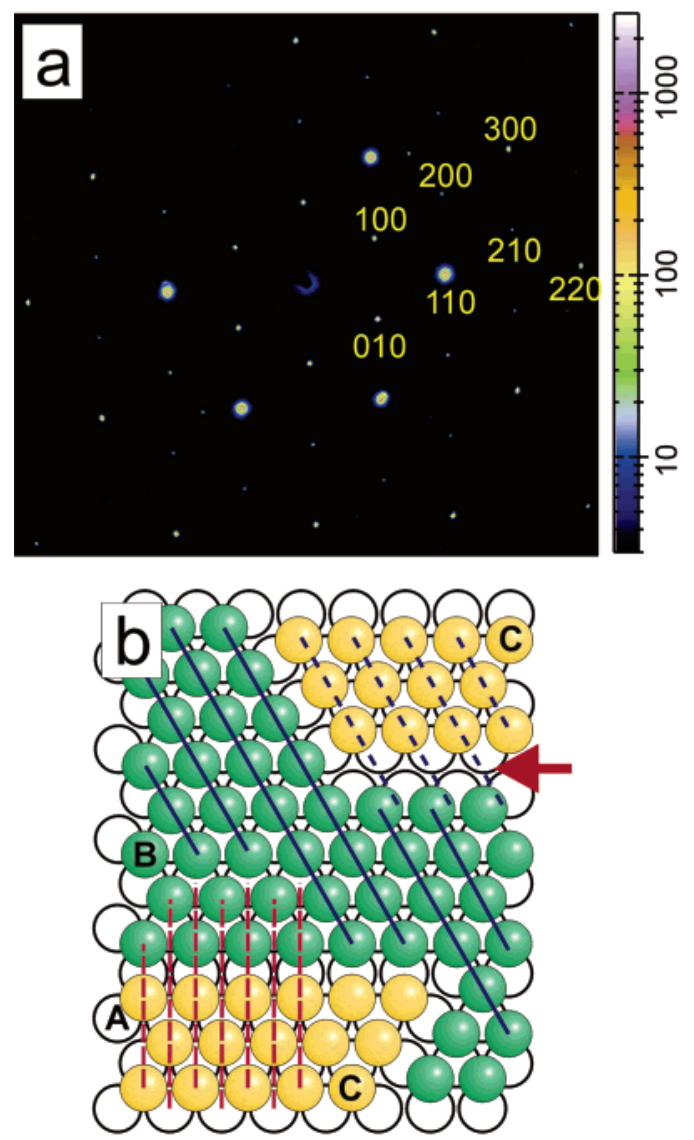

Figure 1. Panel a displays a high-resolution SAXS pattern of hardsphere colloidal crystal measured with $12.5 \mathrm{keV}$ X-ray beam (wavelength 1 Angstrom). The reflections in the left-top corner are absorbed by an acute beamstop. Exposure time is $\tau=1 \mathrm{~s}$. The strong 110 -class reflections saturate the detector, and their width is exaggerated at this exposure time. The Miller indices of several reflections are given. Panel b illustrates the model, which explains the difference in the width of the stacking-dependent and stackingindependent reflections. The long-dashed lines in the bottom-left highlight the periodicity leading to the 110 reflection. The other lines illustrate the periodicity associated to the 100 reflection. The red arrow points onto a broader line defect in between $\mathrm{B}$ and $\mathrm{C}$ domains.

to the Bragg rods, such as the 100 reflection. The lateral width of the Bragg rod should then be related to the inverse of the typical island size.

One way to investigate the existence of stacking islands within the close-packed planes is to move to real space. Microscopy has proved to be an extremely powerful tool to study crystallization, ${ }^{15}$ defect dynamics, ${ }^{16,17}$ and crystal structure formed by sedimentation $^{11,18,19}$ and shear. ${ }^{20}$ The stacking of colloidal crystals has received some attention in these works, but not on individual particle level. The in-plane coexistence of domains with different lateral position has been observed in previous studies (e.g., refs 20-23), but has to date not been quantitatively studied and structurally analyzed. Additionally, microscopy can reveal

(15) Gasser, U.; Weeks, E. R.; Schofield, A.; Pusey, P. N.; Weitz, D. A. Science 2001, 292, 258-262. 323.

(16) Schall, P.; Cohen, I.; Weitz, D. A.; Spaepen, F. Nature 2006, 440, 319-

(17) Schall, P.; Cohen, I.; Weitz, D. A.; Spaepen, F. Science 2004, 305, 19441948.

(18) Elliot, M. S.; Bristol, B. T. F.; Poon, W. C. K. Phys. A 1997, 235, 216 223.

(19) Hoogenboom, J. P.; van Langen-Suurling, A. K.; Romijn, J.; van Blaaderen, A. Phys. Rev. E 2004, 69, 051602 .

(20) Solomon, T.; Solomon, M. J. J. Chem. Phys. 2006, 124
Table 1. Particle and Solvent Properties

\begin{tabular}{lccc}
\hline & $\begin{array}{c}\text { refractive index } \\
\text { difference at } \\
20^{\circ} \mathrm{C}\left(\Delta n_{\mathrm{D}}{ }^{20}\right)\end{array}$ & $\begin{array}{c}\text { density difference } \\
\text { between particles } \\
\text { and medium }\left(\Delta \rho, \mathrm{g} \mathrm{cm}^{-3}\right)\end{array}$ & $\begin{array}{c}\mathrm{Pe}=R^{4} \\
(\Delta \rho \mathrm{g}) /\left(k_{\mathrm{B}} T\right)\end{array}$ \\
\hline SAXS & & 0.92 & $4 \cdot 10^{-4}$ \\
TTC & 0.005 & 0.01 & $3 \cdot 10^{-3}$ \\
cis-decalin & 0.02 & 0.273 & $9 \cdot 10^{-2}$
\end{tabular}

information on the three-dimensional line-defect structure, which cannot be deducted straightforwardly from scattering data.

It is the aim of this work to verify this interpretation of the broadening of the Bragg rods on the basis of real-space confocal microscopy results. We study the occurrence of line-defects in colloidal crystals grown by sedimentation.

\section{Experimental Section}

Experimental Systems. In the SAXS studies presented in Figure 1 and ref 13 sterically stabilized spherical silica colloids were used. The particle diameter was $224 \mathrm{~nm}$ with a polydispersity of $4.1 \%$. The density mismatch between the silica particles and solvent cyclohexane is $0.92 \mathrm{~g} \mathrm{~cm}^{-3}$. Further details can be found in refs 9 and 24. These colloids, which will be referred to as SAXS system in the following, are too small to be used for the confocal microscopy. For the latter we have therefore used poly methyl-metacrylate (PMMA) particles with a diameter of $1.2 \mu \mathrm{m}$, fluorescently labeled with 4-methylamino-ethylmetacrylate-7-nitrobenzo-2-oxa-1,3-diazol (NBD), which were obtained by dispersion polymerization (mass density $\rho=1.17 \mathrm{~g} \mathrm{~mL}^{-1}$, refractive index $\left.n_{\mathrm{D}}=1.50\right){ }^{25}$ The fluorescent dye is covalently incorporated into the polymer network of PMMA, and the particles are sterically stabilized against flocculation by poly(12-hydroxystearic acid). The PMMA particles were $3-5 \%$ polydisperse. This is similar to the polydispersity of the silica spheres used in the SAXS study, which is important since the polydispersity may influence the defect concentration in the crystals. ${ }^{26}$

The particles are dispersed in two apolar solvents: The first solvent was a mixture of tetra chloromethane (Merck "for synthesis"), tetralin (Acros Organics), and cis-decalin (Merck 'for synthesis') (0.35: $0.35: 0.30 \mathrm{v} / \mathrm{v})$. The density of this solvent, as well as the refractive index, nearly matched that of the PMMA particles (see Table 1). Despite the careful density matching, gravity still plays a more important role in comparison to the SAXS system as further discussed in the next section. To understand the effect of the gravity, we additionally used pure $c i$-decalin. These two solvents will be referred to as TTC and De respectively. The use of optically matching apolar solvents $\left(\Delta n_{\mathrm{D}} \approx 0.005\right.$ (TTC) and 0.01 (De)) results in hard sphere interactions. ${ }^{27,28}$

Tuning Gravity. The sedimentation Peclet number, $\mathrm{Pe}=R^{4}(\Delta \rho \mathrm{g}) /\left(\mathrm{k}_{\mathrm{B}} \mathrm{T}\right)$, is usually used to describe the relative importance of the gravitational field. It expresses the ratio between gravitational and thermal energy on the scale of the particle size. Since $\mathrm{Pe} \propto R^{4}$, the Peclet number of the PMMA particles in TTC is significantly larger $\left(\mathrm{Pe} \approx 3 \cdot 10^{-3}\right)$ than the Peclet number of the SAXS crystal during crystallization $\left(\mathrm{Pe} \approx 4 \cdot 10^{-4}\right)$. Further reduction of the gravitational field was not easily attainable. We therefore investigated the effect of gravity by increasing the gravitational

(21) de Villeneuve, V. W. A.; Dullens, R. P. A.; Aarts, D. G. A. L.; Groeneveld, E.; Scherff, J. H.; Kegel, W. K.; Lekkerkerker, H. N. W. Science 2005, 309, $1231-1233$.

(22) Elliot, M. S.; Poon, W. C. K. Adv. Colloid Interface Sci. 2001, 92, 133194.

(23) Poon, W. C. K.; Pusey, P. N. In Observation, Prediction and Simulation of Phase Transitions in complex fluids; Baus, M., Rull, L. F., Ryckaert, J. P., Eds.; Kluwer: Dordrecht, The Netherlands, 1995; pp 3-51.

(24) Petukhov, A. V.; Dolbnya, I. P.; Aarts, D.; Vroege, G. J. Phys. Rev. E 2004, 69.

(25) Bosma, G.; Pathmamanoharan, C.; de Hoog, E. H. A.; Kegel, W. K.; van Blaaderen, A.; Lekkerkerker, H. N. W. J. Colloid Interface Sci. 2002, 245, 292 (26) Pronk, S.; Frenkel, D. J. Chem. Phys. 2004, 120, 6764-6768.

(27) de Hoog, E. H. A.; Kegel, W. K.; van Blaaderen, A.; Lekkerkerker, H. N. W. Phys. Rev. E 2001, 64, 021407

(28) Dullens, R. P. A.; Aarts, D.; Kegel, W. K. Proc. Natl. Acad. Sci. U.S.A 2006, 103, 529-531. 

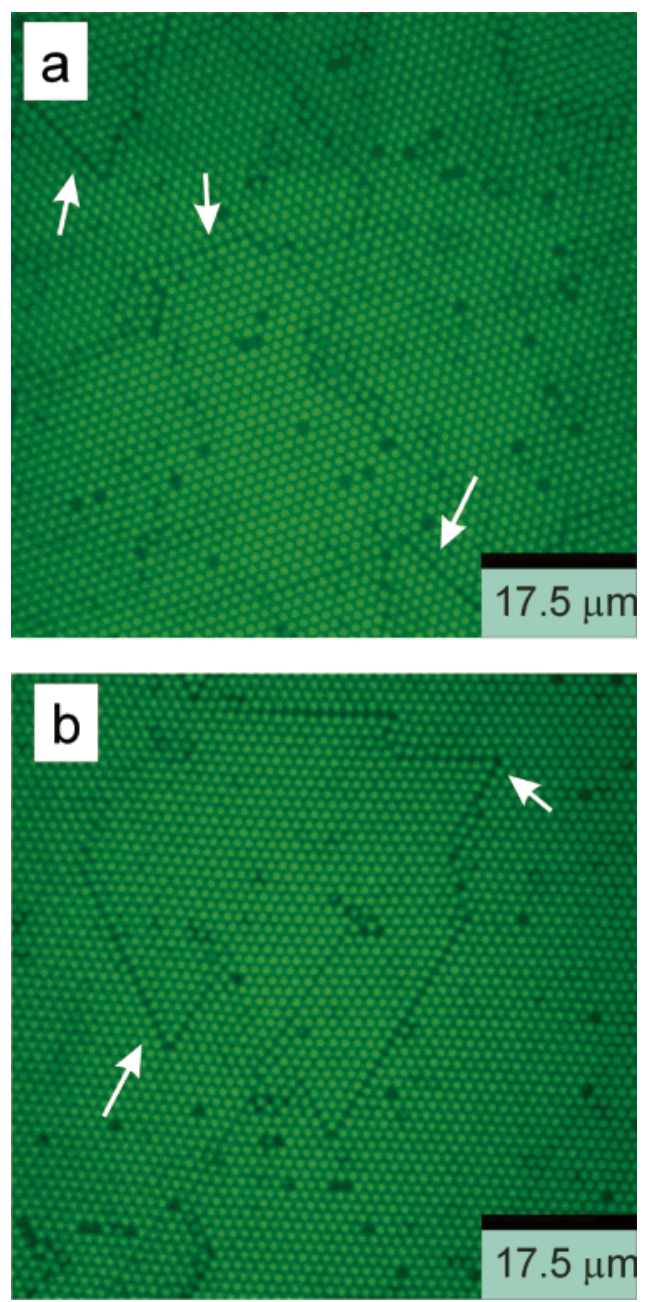

Figure 2. Representative confocal images of both TTC and De crystals. Confocal images of the two different confocal systems for a TTC crystal (a) and a De crystal (b). The white arrows indicate some of the line-defects in between islands with different lateral positions.

field, resulting for $c i s$-decalin in $\mathrm{Pe} \approx 9 \cdot 10^{-2}$. The crystals obtained with the PMMA particles will be further referred to as confocal crystals.

Instrumentation. Thin 2D cross sections of the sample were imaged with a Nikon Eclipse TE2000U laser scanning confocal microscope with a Nikon $\mathrm{C} 1$ scanning head in combination with an argon laser $\left(\lambda_{0}=488 \mathrm{~nm}\right)$ and an oil-immersion lens (Nikon Plan APC 100X, NA 1.4). The $\sim 0.3 \mathrm{~mL}$ samples were stored in small vials. The bottom of the vial was removed and replaced by a microscope cover glass (Chance Propper LTd., West Mids, England, $0.11 \mathrm{~mm}$ thickness), which was glued to the vial using an epoxy glue (Araldit AW2101) with hardener (HW2951).

Experimental Conditions. The suspensions were centrifuged at $500 \mathrm{~g}$, and the supernatant was removed. Assuming that the sediment has a random packing density of $0.66,{ }^{29}$ the samples were redispersed to a certain volume fraction $\varphi_{0}$, and crystals were subsequently formed by sedimentation. By choosing initial volume fractions $\varphi_{0}$ of 0.4 (TTC) and 0.3 (De), the largest crystal grain sizes were obtained. Heterogeneous nucleation at the sample bottom and subsequent upward growth is observed..$^{21}$ Therefore, close to the wall, the crystal structure is solely characterized by the (111) plane, which enables a layer-by-layer investigation of crystal structure. The crystals were imaged from the glass sample bottom to sample heights of $\sim 12 \mu \mathrm{m}$, which corresponds to the first 10 layers of a crystal. Imaging was done within single colloidal crystals by selecting sufficiently large crystallites and by excluding the grain boundaries from the images.

(29) Schaertl, W.; Sillescu, H. J. Stat. Phys. 1994, 77, 1007.

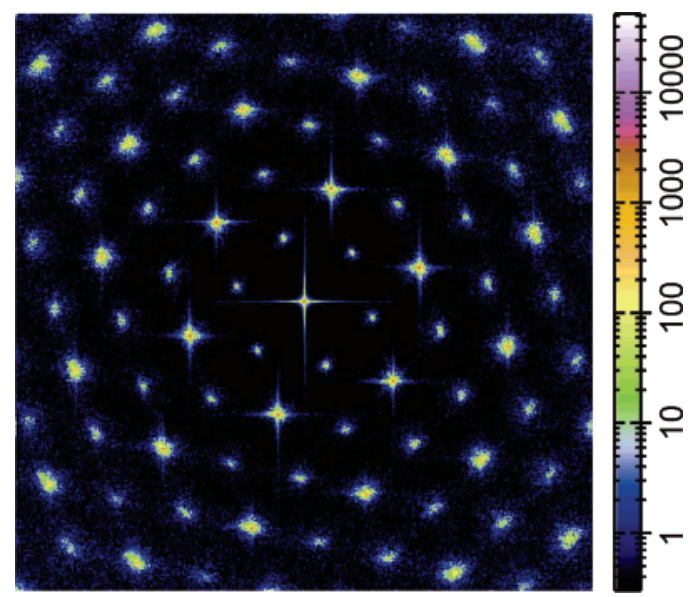

Figure 3. Two-dimensional structure factor pattern $S(\mathbf{q})$ simulated from particle coordinates in a stack of 20 layers in a TTC crystal. The vertical and horizontal stripes are caused by finite-size effects.

Data Analysis. Particle positions were obtained by methods such as those in ref 30; further analysis was performed by $\mathrm{IDL}^{31}$ routines developed at our institute.

\section{Results and Discussion}

Observations. Representative confocal images are shown in Figure 2. Line-defects - and therefore stacking islands-are clearly present. The vast majority of the line-defects are observed to form acute angles of $60^{\circ}$. As shown in Figure $1 \mathrm{~b}$, an obtuse angle of $120^{\circ}$ can only be obtained between line defects of different width. In our images, these wider defect lines are rarely observed, and their length is only on the order of a single particle distance. They result in larger amount of unused crystal space and, therefore, are unfavorable entropically.

Reciprocal Space. In order to determine whether the confocal crystals are analogous to the SAXS crystal, the diffraction patterns of the confocal crystal were simulated. Using $\mathrm{x}, \mathrm{y}$-components of the particle coordinates $r_{i}$, the two-dimensional structure factor $S\left(q_{x}, q_{y}\right)$

$$
S\left(q_{x}, q_{y}\right)=\frac{1}{N} \sum_{i=1}^{N} e^{i\left(q_{x} x_{i}+q_{y} y_{i}\right)}
$$

was calculated. An example of the structure factor pattern is displayed in Figure 3. It is typical for the RHCP crystal structure, and its similarity with the SAXS diffraction pattern is clear. The enhanced visibility of the higher-order reflections in comparison with the experimental result is related to the fact that the particle form factor, which strongly decays with increasing $q$, is not included. The stacking-independent peaks with $h$ - $k$ divisible by 3 are much stronger. At the same time, the peaks with $h-k$ not divisible by 3 are still visible, which is caused by incomplete cancellation of the contributions in a random sequence of stacking positions. We did not observe a remarkable difference in relative widths of the reflections, which might be related to the finite size of the microscope images.

Stacking.We also investigated the stacking of the confocal crystals. To further quantify the stacking disorder, we have determined the stacking parameter $\alpha^{32}$ using three different methods based on the determination of positional or orienta-

(30) Crocker, J. C.; Grier, D. G. J. Colloid Interface Sci. 1996, 179, 298.

(31) Interactive Data Language, Research Systems, Inc.; http://www. ittvis.com (accessed Jan 2007).

(32) Wilson, A. J. C. Proc. Roy. Soc. London, Ser. A 1942, 180, 277. 


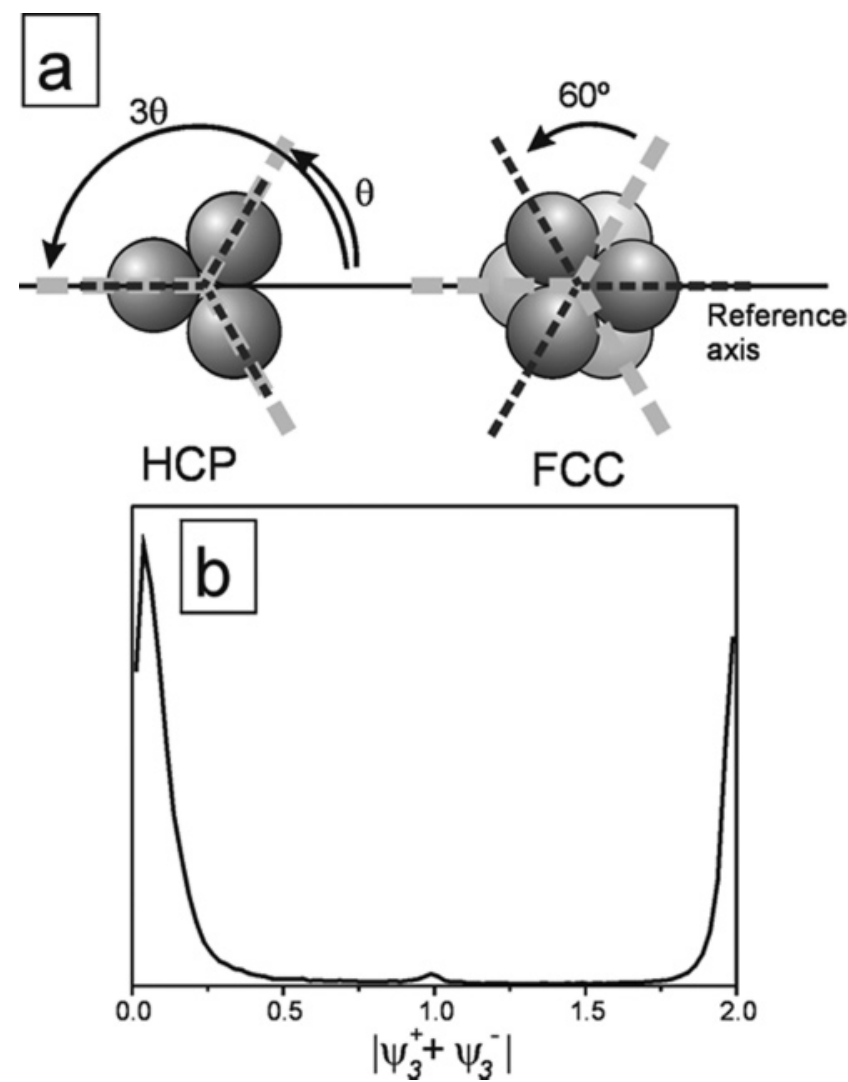

Figure 4. Using the $\psi_{3}$ function to distinguish between HCP and FCC. (a) Schematic top view of a particle in layer $L$ positioned between a layer $L-1$ below, (light gray) and a layer $L+1$ above (dark gray). For FCC stacking of the particle, the $L+1$ layer has rotated $60^{\circ}$ with respect to the $L-1$ layer. The $\psi_{3}$ function uses this shift in orientation to distinguish between FCC and HCP. (b) Typical distribution of $\left|\psi_{3}{ }^{+}+\psi_{3}{ }^{-}\right|$: The first peak at $\left|\psi_{3}{ }^{+}+\psi_{3}{ }^{-}\right| \approx 0$ indicates the particles with FCC stacking, the second peak at $\left|\psi_{3}{ }^{+}+\psi_{3}{ }^{-}\right| \approx 2$ the particles with HCP stacking. The small peak in the middle at $\left|\psi_{3}{ }^{+}+\psi_{3}{ }^{-}\right| \approx 1$ is caused by particles lying above or below a line defect.

tional correlations, as well as a free program ballviewer. ${ }^{33}$ The positional correlation technique compares the particle position distributions of particles separated by one layer in the $z$-direction. All particles $i$ in layer $L+2$ are projected onto the layer $L$ two layers below, and the lateral $x y$-separation of particle $i$ to the nearest particle $j$ in layer $L$ is calculated, resulting in a distance distribution $P_{x y}\left(r_{i j}\right)$. Typical peaks occur at $r_{i j}=0-0.2 a(\mathrm{HCP})$ and $r_{i j}=0.6-0.8 a$ (FCC), with $a=$ the typical inter-particle distance within a crystal. The $\alpha$ parameter is obtained by integration of the peaks.

The orientational correlation method compares the particle orientations of the neighbors of a particle $i$. All particles $i$ in layer $L$ are projected onto the layers $L+1$ (above) and $L-1$ (below), and next neighbors are identified. The relative rotation of the particles in layers $L+1$ and $L-1$ particles toward each other is $60^{\circ}$ for $\mathrm{FCC}$ and $0^{\circ}$ for $\mathrm{HCP}$ (Figure 4 ). To express this rotation, the bond orientational order parameter $\psi_{3}{ }^{ \pm}$is introduced:

$$
\psi_{3}{ }^{ \pm}\left(r_{i}\right)=\frac{1}{N^{ \pm}} \sum_{j^{ \pm}}^{N^{ \pm}} e^{3 i \theta\left(r_{i j}^{ \pm}\right)}
$$

The summation $j^{ \pm}$runs over all $N^{ \pm}$next neighbors in the layer above $\left(\psi_{3}^{+}\right)$or below $\left(\psi_{3}^{-}\right)$of a given particle $i$. The angle

(33) Ackland, G. J.; Jones, A. P. Phys. Rev. B 2006, 73, 054104.
Table 2. Overall Stacking Parameters Obtained by Positional Correlation, Orientational Correlation and Ballviewer

\begin{tabular}{lcccc}
\hline sample & $\begin{array}{c}\text { positional } \\
\text { correlation }\end{array}$ & $\begin{array}{c}\text { orientational } \\
\text { correlation }\end{array}$ & ballviewer & SAXS \\
\hline De & $0.76 \pm 0.05$ & $0.75 \pm 0.06$ & $0.74 \pm 0.06$ & \\
TTC & $0.68 \pm 0.11$ & $0.66 \pm 0.11$ & $0.63 \pm 0.11$ & \\
SAXS $^{34}$ & & & & $0.55 \pm 0.05$
\end{tabular}

between the bond vector connecting the particle with next neighbor $j^{ \pm}$and a fixed arbitrary reference axis is defined as $\theta\left(r_{i j}\right)$. As a result, stacking can now easily be assigned: $\mid \psi_{3}{ }^{+}+$ $\psi_{3}{ }^{-} \mid \approx 0$ and 2 for FCC and HCP, respectively. The stacking parameter then simply is $\alpha=N_{f c c} /\left(N_{f c c}+N_{h c p}\right)$, with $N_{f c c}$ and $N_{h c p}$ being the number of particles with FCC and HCP environment.

Ballviewer distinguishes between particles with HCP, FCC, and $\mathrm{BCC}$ (body-centered cubic) environment from the distribution of angle cosines of all next neighbors.$^{33}$ The stacking parameter was again defined as $\alpha=N_{f c c} /\left(N_{f c c}+N_{h c p}\right)$. We did not use the commonly used kink analysis ${ }^{18}$ since this does not include the possibility of stacking variation within close-packed crystal planes.

The results for the value of the stacking parameter $\alpha$ determined by the three methods are summarized in Table 2: we find $\alpha=$ $0.75 \pm 0.06$ for the De-datasets and $\alpha=0.65 \pm 0.11$ for the TCC-datasets while in the SAXS crystals $\alpha=0.55 \pm 0.05$ as determined by SAXS. ${ }^{34}$ These results indicate that all three colloidal systems yield crystals with RHCP structure. The stacking parameter $\alpha$ displays a tendency toward FCC stacking, which enhances with increasing the role of the gravity. A similar effect of the gravity was reported for slightly charged colloids. ${ }^{11}$ In another study with slightly charged colloids, ${ }^{35}$ the authors find $\alpha=0.4 \pm 0.2$ for $\mathrm{Pe} \approx 10^{-3}$. This is in fact just within the error margins of our TTC measurements, but the deviation might also be related to the limited manual investigation of stacking. The domains of individual lateral positions can be quite large, which can easily affect results obtained by manual inspection. Otherwise, the limited number of crystal layers, charge or perhaps the small grain size-as we will argue in our discussion-can all play a role.

Defect Identification. Confocal images, shown in Figure 2, confirm the existence of islands of different positions in the lateral direction of the crystal. To further analyze the line defect statistics, one needs computer routines for automatic identification of the particles located next to line defects. The unique set of particle distances within a line-defect provides sufficient criteria for identification, which consists of four steps:

The particles within a line-defect, are arranged in unusually spaced rectangles with typical distances of $\sqrt{ }(4 / 3) a \approx 1.15 a$ and $\sqrt{ }(7 / 3) a \approx 1.53 a$. In Figure $5 \mathrm{a}$, a schematic representation of a line-defect and the 2D radial distribution function $g(r)$ are shown

$$
g(r)=\frac{1}{\rho 2 \pi|\vec{r}|}\left\langle\sum_{j \neq i} \delta\left(r-\left|\vec{r}_{j}-\vec{r}_{i}\right|\right)\right\rangle
$$

The small peak in between the first two large peaks in the $g(r)$ confirms the presence of the line-defect distances in the crystal. As a first criterion, particles with neighbors at distances 1.32$1.58 a$ are selected. Note that the larger deviation in $g(r)$ toward lower values results from the significantly larger deviations of

(34) Petukhov, A. V.; Aarts, D. G. A. L.; Dolbnya, I. P.; de Hoog, E. H. A.; Kassapidou, K.; Vroege, G. J.; Bras, W.; Lekkerkerker, H. N. W. Phys. Rev. Lett. 2002, 88, 208301.

(35) Verhaegh, N. A. M.; Vanduijneveldt, J. S.; Vanblaaderen, A.; Lekkerkerker, H. N. W. J. Chem. Phys. 1995, 102, 1416-1421. 


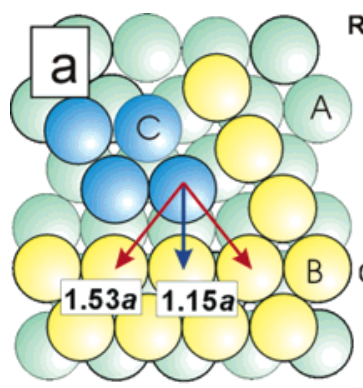

Radial particle distribution function $\mathbf{g}(\mathbf{r})$

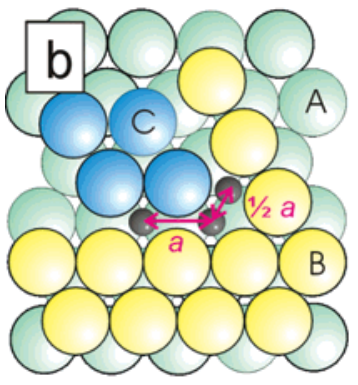

Imaginary particle distance distribution function Pm-im
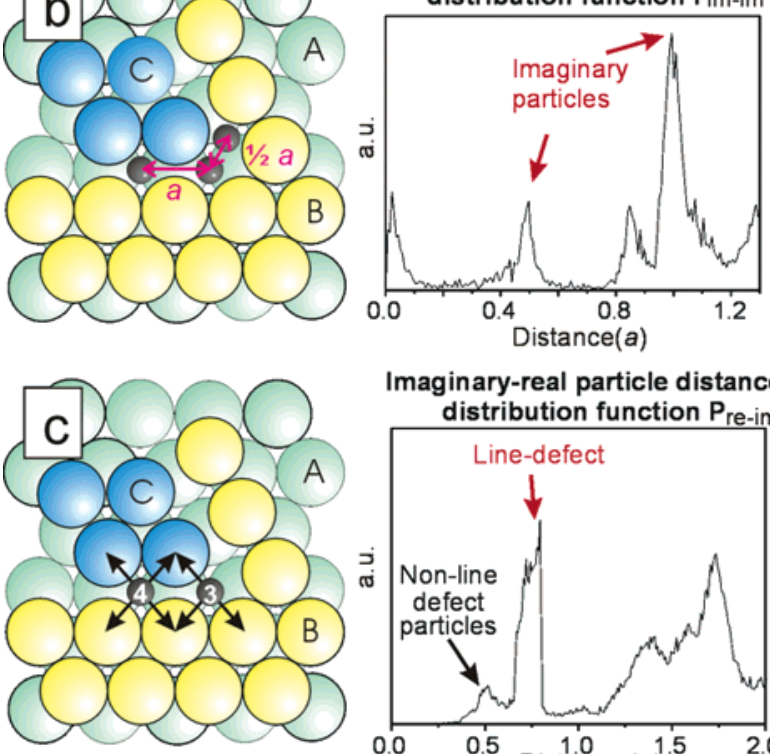

Imaginary-real particle distance distribution function $P_{\text {re-im }}$

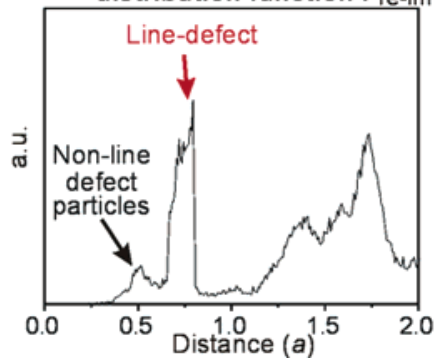

Figure 5. Schematic representations of the unique set of interparticle distances around a line-defect and corresponding 'line-defect radial distribution functions'. (a) The typical line-defect distances. The small peak in the $g(r)$ function confirms the presence of these distances. (b) The imaginary-imaginary interparticle spacings at the corner and along a line-defect, confirmed by the two peaks in $P_{\text {im-im }}$ function (c) Imaginary particle-real particle neighbor distances, confirmed by the second peak in the $P_{\mathrm{im}-\mathrm{re}}$ distribution.

defect particle positions inward than outward the line-defect. To refine the selection, an imaginary particle is placed in between these selected particles (Figure 5b). The coordinates of imaginary particles, which lie within $<0.3 a$, are averaged to one imaginary particle coordinate. In the imaginary particle distance distribution function $P_{\mathrm{im}-\mathrm{im}}\left(r_{i j}\right)$-the pair correlation function of the imaginary particles - peaks occur at $a$ (defect line) and $0.5 a$ (defect corner), as illustrated in Figure $5 \mathrm{~b}$. Therefore, the second criterion requires imaginary particles to have imaginary neighbors at $0.91-1.09 a$, but not at $0.76-0.91 a$, which corresponds to real particles slightly deviated from their ideal lattice position.

The positions of the remaining imaginary particles are compared to the real particle positions, resulting in the imaginary particle $i$-real particle $j$ distance distribution function $P_{\text {re-im }}\left(r_{i j}\right)$ - the pair correlation function of all real particles - imaginary particle combinations (Figure 5c). Its second peak at $\sqrt{7 / 12} a$ represents line-defect particles. The first peak at $\sim 1 / 2 a$ represents nonline-defect particles, which slightly deviated from their ideal lattice positions and were included in the initial selection criteria, but are now excluded here. Each imaginary particle within the line defect should have three or four "real particle" neighbors at this distance. Therefore, the third criterion requires 3 or 4 real next neighbors at $0.60-0.82 a$, and no real next neighbors at
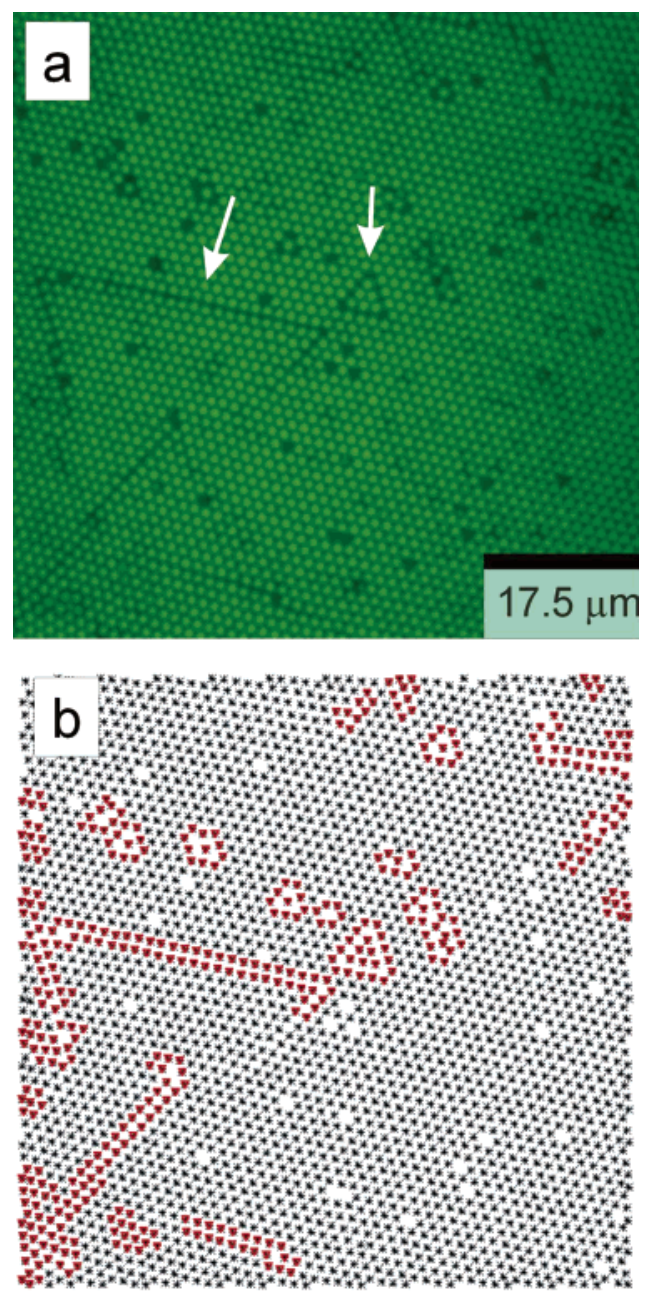

Figure 6. Line-defect identification. (a) Confocal image of the second layer of a TTC crystal; the white arrows indicate the linedefects and islands. (b) The red particles are the particles identified by the four subsequent selection criteria as a line defect particles.

distances smaller than $0.60 a$ for each imaginary particle. A final criterion excludes defect particles next to vacancies as next neighbors: if the amount of "vacancy next neighbors" in the layer equals the amount of vacancy next neighbors found among the defect particles, these are excluded.

The resulting particles are labeled as line defect particles. In Figure 6, the selected line-defect particles are shown: the linedefects are identified with high accuracy using the selection criteria. With the defect particles isolated, the 3D defect structure can be studied. Line-defects that persist in the orthogonal direction of the crystals all stack FCC-like (Figure 7a). This is quite remarkable, since the extent to which a line-defect persists in three dimensions clearly has a large influence on the stacking parameter $\alpha$. Simple geometrical arguments show that $\alpha=n /(n$ +2 ) around a line defect which has FCC stacking, with $n$ being the number of layers in which the line defect occurs (Figure 7b).

For identification purposes, defect particles are projected onto the (111) defect layer below, with typical peaks in the resulting radial distance distribution at $(\sqrt{ } 3)^{-1} a$ and $\sqrt{ }(7 / 3) a$ (Figure $7 \mathrm{c}$ ). Defect particles are identified as caused by the layer below, when particles are found within $1.15 a$ or alternatively at $2.00 a$, the minima after the first and second peak in the distribution function. Defects initiated at the sample bottom are analyzed separately (not presented here), due to lack of structure imposed by a layer below. 

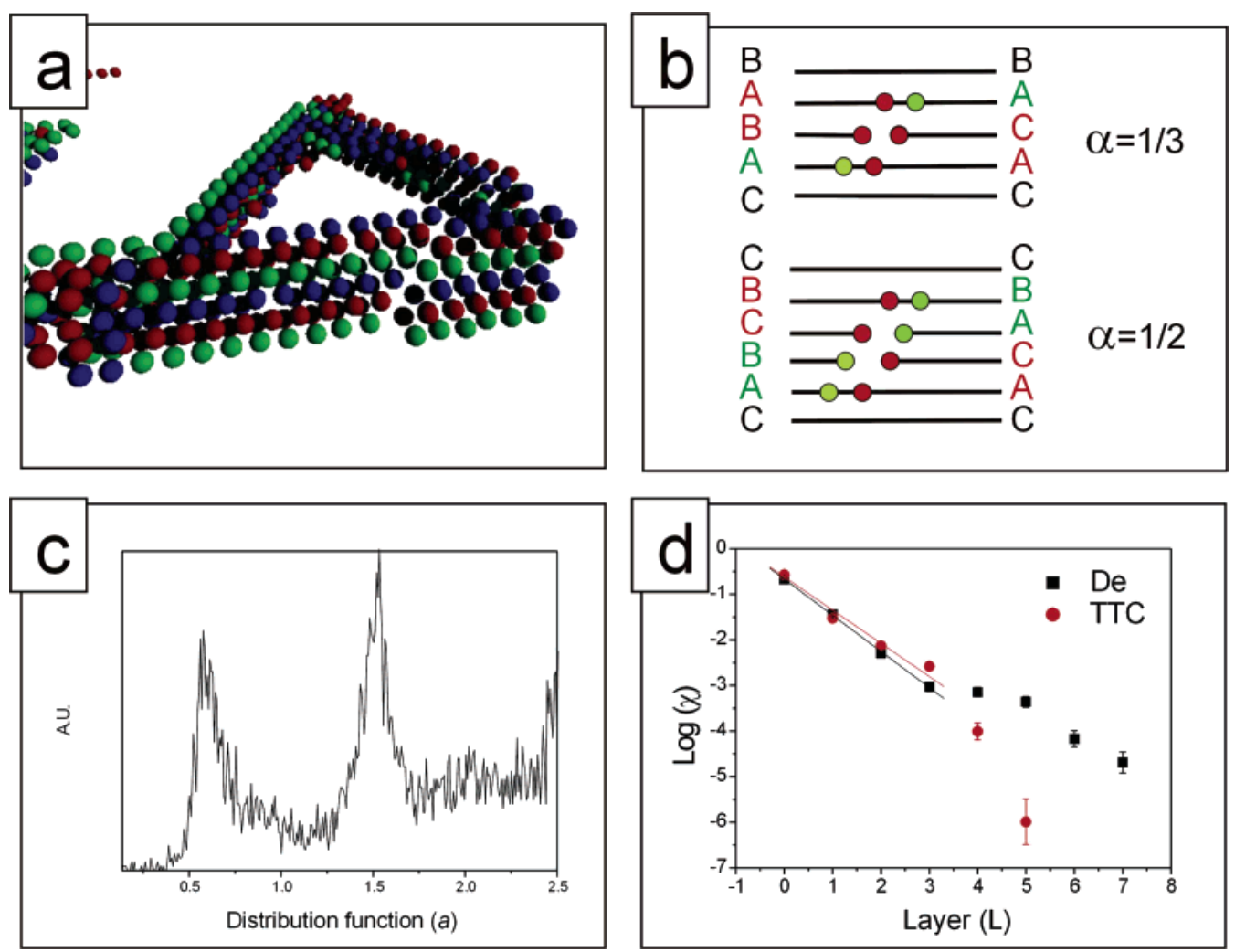

Figure 7. Line-defect in three dimensions. (a) 3D image of line defect particles, green, red, and blue indicate, respectively, A, B, and C positions. FCC-like stacking is clearly visible. (b) The FCC fraction $\alpha$ scales as $n /(n+2)$, for a line-defect present in $n$ consecutive layers. (c) Radial particle distribution of defect particles in subsequent layers. The first peak indicates that the closest defect particles in the subsequent layer are positioned at a lateral position of $(\sqrt{ } 3)^{-1} a$. The second peak occurs at $\sqrt{ }(7 / 3) a$. (d) Defect particle fraction $\chi$ of the defects as a function of the number of layers $L$ in which the defect occurs below a defect particle for De (black squares) and TTC (red circles). The persistence of the defects is fitted to an exponential decay for $L \leq 4$, taking the estimated error bars into account. The plateau for $L>4$, which is especially pronounced in De sample, is attributed to disorder that deforms the crystal lattice.

Table 3. Chance $\zeta$ to Find Another Line-defect Particle in a Subsequent Layer

\begin{tabular}{cccc}
\hline & cutoff distance & De & TTC \\
\hline$\zeta$ & $2.00 \mathrm{a}$ & $0.452 \pm 0.026$ & $0.482 \pm 0.026$ \\
$\zeta$ & $1.15 \mathrm{a}$ & $0.450 \pm 0.018$ & $0.366 \pm 0.027$
\end{tabular}

The number of layers $L$ of defect particles, below every defect particle in the $(L+1)^{\text {th }}$ layer, is determined. The fraction of defect particles in the $(L+1)^{\text {th }}$ layer of a defect is $\chi_{L}=N_{L} / N_{0}$, where $N_{L}$ is the number of defect particles in the $(L+1)^{\text {th }}$ layer and $N_{O}$ is the total number of defect particles. To improve the statistics in the data, the individual datasets are combined for each system. Error bars were included to account for noise in the data, with the error for $N_{L}$ estimated to be $\sqrt{ } N_{L}$, as in a Poisson distribution. We introduce the persistence parameter $\zeta$, which is the chance to find another line defect particle in the layer above a line-defect particle. In Figure $7 \mathrm{~d}, \chi_{L}$ is plotted as a function of $L$, which fits to an exponential decay of the first order with slope $\ln (\zeta)$ for the first few layers. For the different samples, the values of $\zeta$ are given in Table 3. For the De-system the value of $\zeta$ is independent of the used cutoff length value. The sensitivity of the result for TTC might be related to the lower gravity force and osmotic pressure resulting in larger amplitude of particle fluctuations in TTC resulting in slightly less accurate tracking of line-defect particles.

The fact that $\zeta$ is close to $1 / 2$ can be related to the kinetics of the island nucleation and growth. In random-stacking crystals, the new islands are presumably randomly nucleated with one of the two possible lateral positions. The new island that grows toward the existing line defect in the layer below has 50\% chance to have lateral position different from that in both islands below and, therefore, close the defect. This should also be the mechanism by which line-defects anneal out in the initial layers of crystals grown on patterned substrates, with lattice parameters that are slightly off. ${ }^{36}$ Otherwise, by coinciding with the stacking position on the other side of the line-defect, the new island cannot grow further without changing its lateral position. This will result in persistence of the line defect. It is also interesting to understand whether the existence of in-plane stacking disorder yields an effect on the overall stacking probability $\alpha$ in the crystal. For example, a single line defect, which exists only in one layer, necessarily requires an HCP environment for both islands (Figure $5 b)$. On the other hand, an increase of the number of multiple FCC-like stacked line-defects may increase the total number of particles with FCC environment and thus decrease the HCP environment. Furthermore, the persistence of the defects can also be affected by an interaction between stress fields caused by the presence of a defect in several layers and the presence of HCP environments, which are both entropically unfavorable.

Other Defects. An interstitial particle can strongly deform the crystal lattice. Some of the particles present in the vicinity of such a defect will have a position that strongly deviates from the ideal lattice, which makes them difficult for the crystal to resolve within the crystal grain. These defects impose a nonideal lattice for the subsequent layer, in which new defects are likely to occur. In this case the typical $\zeta$ value can be expected to be much higher. Defects that lead to lattice deformations ${ }^{37}$ are present

(36) Lee, W.; Chan, A.; Bevan, M. A.; Lewis, J. A.; Braun, P. V. Langmuir 2004, 20, 5262-5270.

(37) Dullens, R. P. A.; Petukhov, A. V. Europhys. Lett., submitted. 
in our datasets to some extent. Some of them are identified as line-defect with our tracking method. Their presence then easily explains the plateaus that seem to occur in the persistence curves for $L \geq 4$, since the small fraction with higher $\xi$ values should only markedly show up at higher $L$.

\section{Discussion}

Our observations suggest that the three-dimensional line defect structure is FCC-wise stacking. FCC stacked defects do spread the extra line-defect space more homogeneously among the linedefect particles: in a HCP-stacked line defects, particles at either side of the "HCP-kink"18 do not have the same amount of "defect space" available, which should be less favorable entropically. In addition, our samples already contain a larger fraction of FCC stacked particles, which might induce the FCC-wise stacking of line-defects.

In an ideal situation with only three possible particle positions, the line defects either form close loops or run from one side of the image to another. However, the line-defects are also observed to end at dislocations and vacancies, completing the loop through disordered or deformed-lattice positions, which are not identified by our program as line-defects. The polydispersity of the system can explain the abundance of defects observed, and it can (partially) stabilize line defects. For smaller crystallite, line defects are frequently observed to run across a whole grain. It seems that the grain boundaries somehow stabilize the line defects. In small grains, single line defects are mostly observed, which result in a smaller value of the persistence parameter $\zeta$. Also, the stacking parameter $\alpha$ decreases and approaches $1 / 2$. The origin of the stacking disorder could still very well lie in the nucleation and growth kinetics. Whether the stacking islands and resulting line defects are a thermodynamically stable situation for polydisperse spheres or an arrested state likely depends on the extent of polydispersity. Another option-fractionation of particle sizes in different crystal grains ${ }^{38}$ - clearly lies outside the time scale of the present experiment.

\section{Conclusions}

We explain the observed broadening of the small-angle X-ray diffraction stacking-dependent reflections in sedimented hard sphere silica crystals by the presence of island of different lateral positions. These islands result in characteristic line-defects, which we indeed observed in real space in sedimented hard sphere PMMA crystals. The line-defects persist not only in the lateral but also in the orthogonal direction of the crystal, with an FCClike stacking structure. The persistence of the line-defects is an exponential decay, with the persistence parameter $\zeta$, the chance to find another line defect particle in the layer above a linedefect particle, of $\sim 1 / 2$, independent of relative gravity. The fact that $\zeta$ approaches $1 / 2$ is explained by the kinetics of island nucleation and growth, where a new island has $50 \%$ change to close the defect. Also the interaction between the lowest free energy by not having a defect in the subsequent layer and the lowest free energy for the stacking structure (FCC favored over HCP) affects the persistence of the line-defects. Furthermore, defects, which are originated by an interstitial particle, have a higher persistence in the crystal due to the difficulty of the crystal to resolve the defect.

Acknowledgment. It is our pleasure to acknowledge valuable discussions with Willem Kegel, Alan Wouterse, Job Thijssen, Roel Dullens, Jakob Hoogenboom, Anatoly Snigirev, Wim Bras, and Henk Lekkerkerker. The SAXS measurements are performed with help of Maurice Mourad and Esther van den Pol and are made possible due to the technical support of Nicolas Vilayphiou, Kristina Kvashnina, and their colleagues from the DUBBLE beamline. This work was supported by the Stichting voor Fundamenteel Onderzoek der Materie (Foundation for Fundamental Research on matter), which is financially supported by the NWO (Nederlandse Organisatie voor Wetenschappelijk Onderzoek, Dutch Organization for Advancement of Research). The beamtime is provided by the NWO. Support of VWAdV through DFG by SFB TR6 is acknowledged.

\section{LA062966F}

(38) Sear, R. P. Phys. Rev. Lett. 1999, 82, 4244-4247. 